\title{
Uma aplicação do Índice de Desenvolvimento Sustentável aos municípios do estado de Mato Grosso do Sul
}

\author{
A Sustainable Development Index application to Mato Grosso do Sul State \\ municipalities
}

\section{Une application de l'Indice de Développement Durable pour les municipalités état du Mato Grosso do Sul}

\author{
Una aplicación del Índice de Desarrollo Sostenible a los municípios estado de \\ Mato Grosso do Sul \\ Daniel Massen Frainer ${ }^{1}$ \\ Celso Correia de Souza ${ }^{2}$ \\ José Francisco Reis Neto \\ Raul Asseff Castelão ${ }^{3}$
}

Recebido em 10/03/2017; revisado e aprovado em 20/03/2017; aceito em 30/03/2017

DOI: http://dx.doi.org/10.20435/inter.v18i2.1524

\begin{abstract}
Resumo: Este artigo apresenta uma metodologia muito utilizada para o desenvolvimento do Índice de Desenvolvimento Sustentável Municipal (IDSM). O objetivo foi gerar um ranking desses municípios que possa servir como sustentação para a tomada de decisão tanto pública como privada em relação ao desenvolvimento local sustentável. Os resultados apontaram que o principal problema da sustentabilidade está na estrutura institucional que, em certos municípios, encontra-se em estado de alerta.
\end{abstract}

Palavras chave: indicadores; desenvolvimento local; sustentabilidade.

Abstract: This paper presents a method widely used for the development of the Index of Municipal Sustainable Development (IDSM). Aiming to generate a ranking of these cities and serve as an indicator for public decision making as much as private for sustainable local development. The results showed that the main problem of sustainability in this institutional structure that, in some municipalities, is on alert.

Key words: indicators; local development; sustainability.

Résumé: Cet article présente une méthode largement utilisée pour le développement de l'Indice de Développement Municipal Durable (IDSM). Visant à générer un classement de ces villes et servir d'indicateur pour la décision publique faisant autant que privé pour le développement local durable. Les résultats ont montré que le principal problème de la durabilité dans cette structure institutionnelle que dans certaines municipalités est en état d'alerte.

Mots-clés: indicateurs; le développement local; durabilité.

Resumen: Este trabajo presenta un método ampliamente utilizado para el desarrollo del Índice de Desarrollo Municipal Sostenible (IDSM). Con el objetivo de generar un ranking de estas ciudades y servir como un indicador para la toma de decisiones públicas tanto como privada para el desarrollo local sostenible. Los resultados mostraron que el principal problema de la sostenibilidad en esta estructura institucional que en algunos municipios se encuentra en alerta.

Palabras clave: indicadores; desarrollo local; sostenibilidad.

\footnotetext{
${ }^{1}$ Universidade Estadual de Mato Grosso do Sul (UEMS), Campo Grande, Mato Grosso do Sul, Brasil.

${ }^{2}$ Universidade Anhanguera-Uniderp, Campo Grande, Mato Grosso do Sul, Brasil.

${ }^{3}$ Faculdade Salesiana de Santa Tereza (FSST), Corumbá, Mato Grosso do Sul, Brasil.
} 


\section{INTRODUÇÃO}

Indicadores são cruciais para guiar os tomadores de decisão em uma variedade de caminhos de condução de políticas públicas, pois as informações geradas por eles facilitam o processo de decisão e podem ajudar a mensurar o desempenho de políticas voltadas para o desenvolvimento sustentável.

Em 1992, houve uma importante reorganização do rol de indicadores que permitiu aos países criar informações que ajudam nas decisões sobre desenvolvimento sustentável. Essa reorganização a partir da Agenda 21 chama a atenção para o desenvolvimento e a harmonização dos indicadores de desenvolvimento sustentável em nível regional, nacional e global, incluindo a incorporação de um conjunto adequado desses indicadores em comum, regularmente atualizados e acessíveis (NAÇÕES UNIDAS [NU], 2001).

Em resposta a esse alerta expressa na Agenda 21, a Comissão para o Desenvolvimento Sustentável aprovou, em 1995, o programa Work on Indicators of Sustainable Development. O objetivo desse programa foi o de criar indicadores de desenvolvimento sustentável acessíveis aos tomadores de decisão no nível nacional (NU, 2001).

A criação de uma estrutura para organizar a seleção e desenvolvimento de indicadores de sustentabilidade é essencial para a classificação de regiões quanto ao desenvolvimento sustentável e possíveis tomadas de decisão. A necessidade da criação dessa estrutura e a escolha de um conjunto de indicadores podem ser medidas pela prioridade estabelecida de seus usuários, nesse caso, especialistas, sociedade civil e autoridades governamentais, responsáveis pelo desenvolvimento e uso de indicadores para o monitoramento do progresso do desenvolvimento sustentável (NU, 2001).

Além disso, as novas tecnologias promovem uma significativa economia do conteúdo de energia e de recursos naturais no produto das economias modernas, incluindo processos de reciclagem e reprocessamento de recursos naturais, como afirma Buarque (2008).

O presente artigo busca definir quais os temas relevantes do desenvolvimento local no Estado de Mato Grosso do Sul, levando em consideração aspectos da sustentabilidade, construindo um indicador sintético que adequado às especificidades da região. A partir do desenvolvimento recente de indicadores de desenvolvimento sustentável das Nações Unidas e para o Brasil, seria possível avaliar as possibilidades e limitações na comparação dos municípios em relação à situação ideal, fornecendo informações antecipadas de alerta e de prever condições e tendências futuras.

Para isso, o trabalho está dividido em três seções, além desta introdutória. A primeira discute o surgimento e aplicações dos índices de desenvolvimento sustentável no Brasil e no Mundo. A segunda discute uma sugestão de metodologia para o desenvolvimento do Índice de Desenvolvimento Sustentável para os municípios de Mato Grosso do Sul. A seção três analisa os resultados obtidos pela aplicação da metodologia proposta a um conjunto de variáveis representativas dos temas propostos do desenvolvimento local sustentável, classificando os municípios de Mato Grosso do Sul quanto a essa questão.

\section{2 ÍNDICES DE DESENVOLVIMENTO SUSTENTÁVEL NO BRASIL E NO MUNDO}

Em escala nacional e mundial, uma série de indicadores tem surgido, sobretudo ao longo das últimas décadas, incluindo: o Índice de Sustentabilidade Ambiental (ESI); o Índice de Eficiência Ecológico-Econômico, o Índice de Pressão de Consumo; o Índice da "Pegada Ecológica"; o Índice 
de Bem-Estar Econômico Sustentável; o Indicador de Progresso Genuíno e muitos outros, com base em Jollands (2006).

O ponto de partida para uma análise e avaliação do desenvolvimento sustentável está baseado na construção de indicadores proxy para descrever sinteticamente os aspectos do desenvolvimento local considerando aspectos da sustentabilidade, como sugerido por Ronchi et al. (2002), Nourry (2008) e Nader et al. (2008).

Entretanto não há uma forma perfeita ou única de medir o desenvolvimento local sustentável, havendo a necessidade de analisar diferentes indicadores de desenvolvimento e de sustentabilidade para encontrar a melhor forma de quantificá-lo, como afirma Nourry (2008).

Cada indicador, proposto no capítulo 40 da Agenda 21, estabelece uma relação com quatro dimensões primárias do desenvolvimento sustentável: social, econômico, ambiental e institucional. Essas categorias definem a classificação dos indicadores de acordo com a sua dimensão. A organização dessa lista preliminar de indicadores de desenvolvimento sustentável foi sugerida por Bartelmus (1994), em que coloca como um pré-requisito para uma seleção racional de indicadores de desenvolvimento sustentável a discussão conceitual da natureza do desenvolvimento sustentável e a complexa interação entre o ambiente, a economia e a sociedade.

Na versão da Comissão para o Desenvolvimento Sustentável (CSD), o conjunto de indicadores de desenvolvimento sustentável foi desenvolvido entre 1994 a 2001. Esses indicadores foram extensivamente testados, aplicados e usados por muitos países como a base para o desenvolvimento de indicadores nacionais de desenvolvimento sustentável (NU, 2007).

Essas quatro dimensões do Índice de Desenvolvimento Sustentável, representadas em temas relevantes e subtemas que definem as variáveis a serem coletadas na forma de dados para tratamentos estatísticos e posterior definição do Índice de Desenvolvimento Sustentável, formam a estrutura para esse cálculo e se apresentam conforme quadro 1.

\begin{tabular}{|l|l|}
\hline \multicolumn{1}{|c|}{ Social } & \multicolumn{1}{c|}{ Ambiental } \\
\hline Educação & Recursos hídricos \\
\hline Emprego & Agricultura \\
\hline Saúde & Zona costeira \\
\hline Moradia & Ambiente marinho \\
\hline Qualidade de vida & Pesca \\
\hline Distribuição de renda & Poluição do ar \\
\hline Crime & Mudanças globais no clima \\
\hline População & Uso sustentável dos recursos naturais \\
\hline Valores éticos e morais & Turismo sustentável \\
\hline Papel das mulheres & Uso da terra \\
\hline Acesso a terra e recursos & \\
\hline Estrutura da comunidade & \\
\hline Exclusão social & \\
\hline & \\
\hline Dependência econômica & Integração das tomadas de decisão \\
\hline Energia & Capacitação \\
\hline Consumo e padrões de produção & Ciência e tecnologia \\
\hline Manejo dos resíduos & Sensibilização e informação pública \\
\hline Transportes & Convenções internacionais e cooperação \\
\hline Mineração & Governança \\
\hline Estrutura econômica e desenvolvimento & Estruturas institucionais e legislativas \\
\hline Comércio & Preparaçãoo para desastres \\
\hline Produtividade & Participação pública \\
\hline
\end{tabular}

Quadro 1 - Dimensões e principais temas para a estrutura de Indicadores de Desenvolvimento Sustentável

Fonte: Nações Unidas (2001). 
Alguns dos indicadores do quadro 1 já foram testados em diversos países, e publicados como iniciativas de indicadores de desenvolvimento sustentável aplicados por Organismos Internacionais, conforme Hak (2007).

A estrutura dos indicadores deve levar em consideração, dentro de cada dimensão, temas que possam selecionar um número mínimo de indicadores para que um determinado tema possa ser explorado. Países são encorajados pelas Nações Unidas a adotar e usar um conjunto de indicadores, daqueles propostos, como ponto de partida para seus programas de indicadores nacionais (NU, 2001).

Nesse contexto, a construção de indicadores de desenvolvimento sustentável no Brasil foi ao encontro dos esforços internacionais para concretização das ideias e dos princípios formulados na Conferência das Nações Unidas sobre Meio Ambiente e Desenvolvimento (Rio 92), realizada em 1992.

Com a publicação de indicadores de desenvolvimento sustentável, o Instituto Brasileiro de Geografia e Estatística (IBGE) inicia a pesquisa sequencialmente, de dois em dois anos, sobre esse tema no país. O conjunto de informações disposto sobre a realidade brasileira, em suas dimensões ambiental, social, econômica e institucional cria uma contribuição seminal para os tomadores de decisão sobre o panorama abrangente dos principais temas relacionados ao desenvolvimento sustentável no Brasil.

Em sua última edição, em 2014, os Indicadores de Desenvolvimento Sustentável do IBGE foram desenvolvidos a partir de 60 indicadores que, em sua maior parte, se dividem pelas quatro dimensões, permitindo o acompanhamento dos fenômenos ao longo do tempo e o exame de sua ocorrência no território nacional, conforme IBGE (2014).

Entretanto, para definir um recorte estadual ou municipal, o número de indicadores ficaria mais restrito. Diferente da magnitude de indicadores disponíveis no âmbito nacional, no nível regional boa parte destes são realizados de forma esporádica ou decenal, em períodos de censo populacional. Nas pesquisas amostrais ou realizadas de forma sistemática nos estados e municípios, os dados disponíveis restringem o número de indicadores que podem ser utilizados.

De acordo Roldán e Saldívar-Valdés (2002), a metodologia proposta para a seleção do conjunto de indicadores locais para comparar e gerar um ranking dos municípios de uma região do México utiliza como critério para a seleção os seguintes requisitos:

1) A disponibilidade e confiabilidade das fontes de dados;

2) A estatística de dados mais atualizada possível;

3) A representação na análise de três sistemas: natural, social e econômico, com sua importância regional;

4) Uma aproximação holística que inclua termos quantitativos e qualitativos.

A metodologia proposta neste artigo deve considerar todos os municípios de Mato Grosso do Sul, incluindo todas as dimensões propostas pela Comissão para o Desenvolvimento Sustentável e utilizando a metodologia do Índice de Desenvolvimento Sustentável (IDS), desenvolvido em nível nacional pelo IBGE. Isso torna a análise mais completa, podendo-se comparar os indicadores regionais propostos aos indicadores nacionais.

Os indicadores selecionados a partir dos temas descritos no quadro 1 dão origem à construção metodológica do Índice de Desenvolvimento Sustentável Municipal (IDSM), para os municípios do Estado de Mato Grosso do Sul. 


\section{MATERIAIS E MÉTODOS}

A metodologia proposta para elaboração do sistema do IDSM foi a de avaliar os níveis de sustentabilidade, considerando os critérios mundialmente utilizados para a escolha dos indicadores de sustentabilidade e as especificidades de enfoque no desenvolvimento local. Para Martins e Cândido (2008), ao considerar cada um dos indicadores selecionados, deve-se atentar às seguintes características deles: a) ser significativo para a realidade investigada e para o enfoque do estudo; b) ser relevante para as decisões que orientam as políticas públicas; c) refletir as mudanças temporais; d) permitir um enfoque integrado e sistêmico; e) utilizar variáveis mensuráveis; f) ser de fácil interpretação e comunicação; e g) ter uma metodologia bem definida, transparente e objetiva aos propósitos da investigação.

A seleção de indicadores proposta neste artigo foi realizada em três etapas: a) construção de um banco de dados municipal para questões do desenvolvimento sustentável, selecionando os temas dentro de cada uma das dimensões do IDS; b) definição de indicadores a padronização das variáveis para torná-las comparáveis e passíveis de agregação em um indicador sintético dentro de cada uma das dimensões; c) resultados obtidos para os municípios dos indicadores por dimensões e classificados gerando um ranking através dos resultados obtidos pelo IDSM.

Essa primeira etapa de seleção dos temas para gerar um banco de dados municipal obedeceu às metodologias nacionais, considerando as variáveis relevantes, dentro de cada dimensão, que possuía informações municipais.

Para isso, buscou-se utilizar a mesma fonte e ano para os indicadores. As informações coletadas estão disponíveis em várias fontes, a saber: ANDA (2013), ENERSUL (2013), IBGE (2013), IPEA (2013), SANESUL (2013), Brasil (2013), DETRAN (2013), relativos aos dados do ano de 2010, apresentados por dimensão no quadro 2.

\begin{tabular}{|c|l|c|c|}
\hline Dimensão & \multicolumn{1}{|c|}{ Indicador } & Fonte & Ano \\
\hline \multirow{5}{*}{ Social } & Índice de Gini da renda & IBGE & 2010 \\
& Razão da renda & IBGE & 2010 \\
& Taxa de desemprego & IBGE & 2010 \\
& Taxa de pobreza & IBGE & 2010 \\
& Renda média per capita & IBGE & 2010 \\
& Analfabetismo & IBGE & 2010 \\
& Esperança de vida & IPEA & 2013 \\
& Mortalidade infantil & IPEA & 2013 \\
& IDHm frequência escolar & IPEA & 2013 \\
& IDHm latência escolar & IPEA & 2013 \\
& Taxa de homicídios & IBGE & 2010 \\
& Taxa crescimento populacional & IBGE & 2010 \\
\hline \multirow{5}{*}{ Econômica } & PIB per capta & IBGE & 2010 \\
& Finanças municipais & Tesouro Nacional & 2010 \\
& Consumo de energia & ENERSUL & 2010 \\
& Intensidade de energia & IBGE/ENERSUL & 2010 \\
& Taxa de investimento & Tesouro Nacional & 2010 \\
\hline & Água encanada & Sanesul & 2010 \\
& Saneamento & IPEA & 2010 \\
& Frota veículos & DETRAN/MS & 2010 \\
& Consumo de combustíveis fósseis & Distribuidores & 2010 \\
& Uso de fertilizantes & ANDA & 2008 \\
\hline \multirow{5}{*}{ Ambiental }
\end{tabular}

INTERAÇÕES, Campo Grande, MS, v. 18, n. 2, p. 145-156, abr./jun. 2017. 


\begin{tabular}{|c|l|c|c|}
\hline Dimensão & \multicolumn{1}{|c|}{ Indicador } & Fonte & Ano \\
\hline \multirow{5}{*}{ Institucional } & IBGE & 2010 \\
& Domićílios com telefone fixo & IBGE & 2010 \\
& Domicílios com telefone celular & IBGE & 2010 \\
& Domićílios com internet & MEC & 2010 \\
& Matriculados na universidade & IBGE & 2010 \\
& Qualificação consolidada & IBGE & 2010 \\
\hline
\end{tabular}

Quadro 2 - Lista de indicadores usados no presente artigo

Fonte: Elaborado pelos autores.

Diferente do índice nacional, que trabalha com informações coletadas sistematicamente, no indicador municipal deve-se fazer algumas transformações de dados para facilitar a compreensão dos resultados e classificação dos municípios. Sendo assim, a compilação de dados e o desenvolvimento de indicadores que forneçam informações simples e comparáveis para portes diferentes de municípios se mostrou necessária.

Uma vez realizada a primeira etapa de seleção de indicadores, passa-se para a padronização das variáveis selecionadas pelo método sugerido por Waquil et al. (2010) transformando os indicadores em índices, permitindo a comparabilidade de variáveis de unidades distintas além de normalizar os dados em um número que varia de 0 a 1 , de modo que, quanto mais próximo de 1 , melhor se apresenta o município em relação às questões que envolvem a sustentabilidade. Sendo assim, a escala de valores de mínimo e máximo corresponde, respectivamente, a 0 (insustentabilidade) e 1 (sustentabilidade).

Nessa perspectiva, deve-se ainda levar em consideração que existem indicadores que são positivamente correlacionados com a sustentabilidade, e outros, negativamente. Para realizar uma agregação, todos os índices devem apontar para uma relação positiva com relação à sustentabilidade para poderem ser agregados gerando um indicador sintético. Sendo assim, a relação (positiva ou negativa) que essas variáveis apresentam com a sustentabilidade, identifica-se pela seguinte relação: positivas (quanto maior melhor e quanto menor pior) e negativas (quanto menor melhor e quanto maior pior), conforme o contexto de suas relações.

O procedimento para padronização prevê que, se o indicador tem influência positiva ou negativa sobre a sustentabilidade, deveria ser analisado separadamente conforme as equações (1) e (2), respectivamente. Teoricamente, para um indicador positivo, em (1), o valor observado máximo terá valor 1 como escore, isto é, quanto maior o indicador, melhor será índice e, quanto menor o indicador, pior será o índice. Já para o indicador negativo, quanto maior o indicador, pior será o índice e, quanto menor o indicador, melhor será o índice. Utilizando a equação (2) o seu comportamento será como aquele do indicador positivo, isto é, quanto maior, melhor (valor máximo 1), e quanto menor, pior (valor mínimo zero).

$$
\begin{aligned}
& \mathrm{x}_{(+)}=\frac{\text { obs }- \text { mín }}{\text { máx }- \text { mín }} \\
& x_{(-)}=\frac{\text { máx }- \text { obs }}{\text { máx }- \text { mín }}
\end{aligned}
$$


Onde:

$X_{(.)}=$indicador padronizado, calculado para cada município; obs = valor do indicador em cada município; mín = valor mínimo do indicador de todos os municípios; máx = valor máximo do indicador de todos os municípios.

O valor mínimo e o valor máximo de cada indicador em estudo foram atribuídos conforme cada variável selecionada, não importando a sua unidade de medida. Desse modo, foi possível normalizar os dados para uma base comparável.

Uma vez realizada a padronização dos valores dos indicadores para todos os municípios do MS, foram realizadas agregações dos índices gerados nas quatro dimensões definidas: social, econômica, ambiental e institucional.

O valor do índice de cada dimensão $\mathrm{j}(\mathrm{j}=1,2, \cdots, \mathrm{m})$, foi encontrado pela média aritmética simples dos índices de cada um dos temas i $(i=1,2, \cdots, n)$, que compõe a dimensão analisada. Foram estabelecidos pesos iguais para as variáveis dentro de cada dimensão. A expressão (3) é a média aritmética simples dos índices representativos de cada dimensão.

$D_{j}=\frac{\sum_{i=1}^{n} T_{i}}{n} ; \quad \mathrm{j}=12,3 \cdots, \mathrm{m}$

Onde:

$I D_{j}=$ índice da dimensão $\mathrm{j}(\mathrm{i}=1,2, \ldots, \mathrm{n})$;

$T_{i}=$ índice $i$

$\mathrm{m}=$ número de dimensões;

$n=$ número de índices.

A fórmula final do IDSM foi gerada pela agregação através da média aritmética simples dos seus índices temáticos, expressão (4).

$I D S M=\frac{I D S+I D E+I D I+I D A}{4}$

Onde:

IDS = índice da dimensão social; IDE = índice da dimensão econômica; IDI = índice da dimensão institucional e; IDA = índice da dimensão ambiental.

Todos os índices gerados para cada dimensão e o índice final IDSM podem ser classificados quanto ao nível de sustentabilidade, gerando um ranking dos municípios do Estado. Na tabela 1 estão explicitados os intervalos do IDSM e que podem também ser utilizados para analisar individualmente os índices de cada dimensão em termos de níveis de sustentabilidades para os municípios, utilizando a classificação sugerida por Martins e Cândido (2008). 
Tabela 1 - Classificação do nível de sustentabilidade dos municípios

\begin{tabular}{lc}
\hline Índice $(\mathbf{0}-\mathbf{1})$ & Nível de sustentabilidade \\
\hline $0,0000-0,2500$ & Crítico \\
$0,2501-0,5000$ & Alerta \\
$0,5001-0,7500$ & Aceitável \\
$0,7501-1,0000$ & Ideal \\
\hline
\end{tabular}

Fonte: Adaptado para a pesquisa a partir de Martins e Cândido (2008).

Dessa forma, os municípios de Mato Grosso do Sul, quando produzidos os resultados dos índices, podem ser classificados individualmente ou como um todo, fornecendo uma base de comparabilidade entre os municípios em determinado ano, ou fornecendo uma análise ao longo do tempo do mesmo município.

Segundo a tabela 1, os índices de sustentabilidade com valores entre 0,0000 e 0,2500 são caracterizados pelo desempenho que revela um estado crítico de sustentabilidade; os índices com valores entre 0,2501 e 0,5000 foram representados pelo desempenho que revela um estado ou situação de alerta; os índices com valores entre 0,5001 e 0,7500 foram representados pelo desempenho que revela um estado ou situação aceitável e; por último, os índices com valores entre 0,7501 e 1,0000 foram representados pelo desempenho que revela estado ou situação ideal de sustentabilidade.

\section{RESULTADOS OBTIDOS PELO IDSM PARA OS MUNICÍPIOS DE MATO GROSSO DO SUL}

A partir do cálculo de cada índice (IDS, IDE, IDA e IDI), para cada município do Estado de Mato Grosso do Sul, foi realizada uma agregação mediante a média aritmética simples desses índices para a obtenção do IDSM para cada município do Estado, estabelecendo um ranking dos com situação aceitável e de alerta, sendo os primeiros apresentados na Tabela 2.

Tabela 2 - Resultados ranking em situação aceitável para o IDSM

\begin{tabular}{lccccc}
\hline Municípios & IDS & IDE & IDA & IDI & IDSM \\
\hline Campo Grande & 0,7838 & 0,5608 & 0,6092 & 0,8272 & 0,6953 \\
Dourados & 0,7123 & 0,5816 & 0,6446 & 0,7728 & 0,6778 \\
Três Lagoas & 0,7442 & 0,6687 & 0,5359 & 0,6786 & 0,6568 \\
Chapadão do Sul & 0,8495 & 0,6740 & 0,4557 & 0,6009 & 0,6450 \\
São Gabriel do Oeste & 0,7543 & 0,6589 & 0,6273 & 0,4601 & 0,6251 \\
Maracaju & 0,7367 & 0,5925 & 0,5672 & 0,5117 & 0,6020 \\
Corumbá & 0,5940 & 0,7060 & 0,5425 & 0,5250 & 0,5919 \\
Costa Rica & 0,6947 & 0,6426 & 0,6132 & 0,3924 & 0,5857 \\
Nova Andradina & 0,7169 & 0,5250 & 0,5436 & 0,5400 & 0,5814 \\
Alcinópolis & 0,7402 & 0,5369 & 0,7486 & 0,2884 & 0,5785 \\
Paranaíba & 0,6789 & 0,4695 & 0,5846 & 0,5616 & 0,5736 \\
Cassilândia & 0,6347 & 0,4772 & 0,5639 & 0,6163 & 0,5731 \\
Bataguassu & 0,6919 & 0,6145 & 0,6089 & 0,3756 & 0,5728 \\
Vicentina & 0,6783 & 0,5670 & 0,6603 & 0,3633 & 0,5673 \\
Figueirão & 0,6292 & 0,5259 & 0,8490 & 0,2331 & 0,5593 \\
Rio Brilhante & 0,7003 & 0,6535 & 0,5461 & 0,3364 & 0,5591 \\
Selvíria & 0,4493 & 0,4763 & 0,6695 & 0,6401 & 0,5588 \\
Brasilândia & 0,6306 & 0,6067 & 0,6621 & 0,3105 & 0,5525 \\
Ivinhema & 0,7264 & 0,4941 & 0,5170 & 0,4657 & 0,5508
\end{tabular}




\begin{tabular}{lccccc}
\hline Municípios & IDS & IDE & IDA & IDI & IDSM \\
\hline Naviraí & 0,6065 & 0,5221 & 0,5917 & 0,4685 & 0,5472 \\
Glória de Dourados & 0,6679 & 0,4147 & 0,5604 & 0,5124 & 0,5389 \\
Inocência & 0,6570 & 0,5988 & 0,6226 & 0,2740 & 0,5381 \\
Aquidauana & 0,6029 & 0,4147 & 0,6246 & 0,5099 & 0,5380 \\
Jardim & 0,6605 & 0,4238 & 0,6134 & 0,4404 & 0,5345 \\
Jateí & 0,6453 & 0,4882 & 0,6883 & 0,3145 & 0,5341 \\
Caarapó & 0,5781 & 0,6015 & 0,6098 & 0,3412 & 0,5327 \\
Rochedo & 0,6237 & 0,5207 & 0,7724 & 0,2075 & 0,5311 \\
Bandeirantes & 0,6196 & 0,5735 & 0,6698 & 0,2556 & 0,5296 \\
Aral Moreira & 0,4853 & 0,6413 & 0,7533 & 0,2315 & 0,5279 \\
Sonora & 0,5687 & 0,6140 & 0,6196 & 0,3070 & 0,5273 \\
Aparecida do Taboado & 0,6121 & 0,5118 & 0,6251 & 0,3445 & 0,5234 \\
Ladário & 0,6270 & 0,3637 & 0,6272 & 0,4692 & 0,5218 \\
Fátima do Sul & 0,6311 & 0,4029 & 0,5770 & 0,4697 & 0,5202 \\
Ponta Porã & 0,5468 & 0,4608 & 0,6097 & 0,4604 & 0,5194 \\
Água Clara & 0,5977 & 0,6300 & 0,5914 & 0,2535 & 0,5181 \\
Deodápolis & 0,5991 & 0,4815 & 0,6211 & 0,3577 & 0,5149 \\
Camapuã & 0,5968 & 0,4956 & 0,6424 & 0,3203 & 0,5138 \\
Taquarussu & 0,5408 & 0,4583 & 0,7515 & 0,2929 & 0,5109 \\
Ribas do Rio Pardo & 0,6016 & 0,5858 & 0,5865 & 0,2638 & 0,5095 \\
Mundo Novo & 0,5374 & 0,4608 & 0,5904 & 0,4437 & 0,5081 \\
Laguna Carapã & 0,5975 & 0,5396 & 0,6288 & 0,2646 & 0,5076 \\
Eldorado & 0,5354 & 0,5510 & 0,5906 & 0,3494 & 0,5066 \\
\hline
\end{tabular}

Fonte: Resultados obtidos a partir dos dados da pesquisa.

Os municípios de Campo Grande, Dourados e Três Lagoas são os primeiros do ranking, apresentando índices aceitáveis para todos os indicadores e para o IDSM. Embora os demais municípios estejam em nível aceitável de sustentabilidade no conjunto dos indicadores, alguns apresentam situações críticas em alguns indicadores, individualmente. Por exemplo, os municípios de Selvíria e Aral Moreira apresentam para o indicador social valores considerados de alerta.

Para os municípios de Paranaíba, Cassilândia, Selvíria, Ivinhema, Glória de Dourados, Aquidauana, Jardim, Jateí, Ladário, Fátima do Sul, Ponta Porã, Deodápolis, Camapuã, Mundo Novo e Taquarussu, o mesmo ocorre para o indicador econômico, em que podem ser classificados como em situação de alerta.

Já os municípios de São Gabriel do Oeste e Chapadão do Sul apresentam IDSM aceitável, mas apresentam indicadores ambientais e institucionais, respectivamente, em estado de alerta. Os municípios que obtiveram índices de sustentabilidade em estado de alerta estão listados na tabela 3:

Tabela 3 - Resultados ranking em situação de alerta para o IDSM

\begin{tabular}{lccccc}
\hline Município & IDS & IDE & IDA & IDI & IDSM \\
\hline Bonito & 0,6405 & 0,4073 & 0,6272 & 0,3208 & 0,4989 \\
Corguinho & 0,5466 & 0,5049 & 0,7375 & 0,2051 & 0,4985 \\
Sidrolândia & 0,5996 & 0,4733 & 0,6126 & 0,3071 & 0,4982 \\
Pedro Gomes & 0,5292 & 0,5092 & 0,6728 & 0,2754 & 0,4966 \\
Porto Murtinho & 0,5342 & 0,5382 & 0,6728 & 0,2315 & 0,4942 \\
Coxim & 0,6454 & 0,4839 & 0,4318 & 0,4131 & 0,4936 \\
Bela Vista & 0,6055 & 0,4138 & 0,6314 & 0,3233 & 0,4935 \\
Bodoquena & 0,4948 & 0,4718 & 0,6722 & 0,3315 & 0,4926
\end{tabular}




\begin{tabular}{lccccc}
\hline Município & IDS & IDE & IDA & IDI & IDSM \\
\hline Batayporã & 0,5473 & 0,5219 & 0,5909 & 0,3093 & 0,4924 \\
Caracol & 0,6334 & 0,4404 & 0,7053 & 0,1898 & 0,4922 \\
Terenos & 0,6018 & 0,4867 & 0,6607 & 0,2125 & 0,4904 \\
Anastácio & 0,5173 & 0,4341 & 0,6576 & 0,3515 & 0,4901 \\
Iguatemi & 0,4915 & 0,5195 & 0,6254 & 0,3161 & 0,4881 \\
Rio Verde de MT & 0,6182 & 0,4383 & 0,5855 & 0,2954 & 0,4844 \\
Itaporã & 0,5259 & 0,4891 & 0,5848 & 0,3341 & 0,4835 \\
Amambai & 0,5401 & 0,4204 & 0,5757 & 0,3794 & 0,4789 \\
Antônio João & 0,4280 & 0,5708 & 0,6492 & 0,2601 & 0,4770 \\
Anaurilândia & 0,5776 & 0,4592 & 0,6099 & 0,2556 & 0,4756 \\
Rio Negro & 0,6301 & 0,3364 & 0,6379 & 0,2875 & 0,4730 \\
Guia Lopes da Laguna & 0,4970 & 0,4394 & 0,6210 & 0,2932 & 0,4626 \\
Juti & 0,4272 & 0,4741 & 0,6991 & 0,2101 & 0,4526 \\
Novo Horizonte do Sul & 0,5586 & 0,5432 & 0,4652 & 0,2307 & 0,4494 \\
Douradina & 0,5678 & 0,3036 & 0,6151 & 0,2941 & 0,4452 \\
Dois Irmãos do Buriti & 0,4065 & 0,3734 & 0,7233 & 0,2628 & 0,4415 \\
Itaquiraí & 0,3854 & 0,5626 & 0,5830 & 0,2109 & 0,4355 \\
Sete Quedas & 0,5581 & 0,4812 & 0,6368 & 0,0579 & 0,4335 \\
Nioaque & 0,4756 & 0,3896 & 0,6547 & 0,2121 & 0,4330 \\
Jaraguari & 0,6207 & 0,4058 & 0,5110 & 0,1719 & 0,4273 \\
Miranda & 0,4220 & 0,3972 & 0,5717 & 0,2428 & 0,4084 \\
Coronel Sapucaia & 0,3394 & 0,3393 & 0,7104 & 0,1959 & 0,3962 \\
Tacuru & 0,2868 & 0,4016 & 0,6492 & 0,1613 & 0,3747 \\
Paranhos & 0,3740 & 0,3017 & 0,5980 & 0,1545 & 0,3571 \\
Japorã & 0,1822 & 0,2974 & 0,6467 & 0,0793 & 0,3014 \\
\hline Fan & & & & \\
\hline
\end{tabular}

Fonte: Resultados obtidos a partir dos dados da pesquisa.

Observa-se que todos os municípios com nível de sustentabilidade de alerta, com valor abaixo de 0,50, apresentam situação crítica na dimensão institucional. O grande problema para o tomador de decisão institucional seria, a partir desse diagnóstico, definir estratégias para meIhorar a estrutura de oferecimento de: qualificação de mão-de-obra e investimento em ciência e tecnologia; melhoria da estrutura de atendimento dos serviços públicos à população em geral e; política de incentivo para a implantação de mais unidades de apoio à pesquisa.

\section{CONSIDERAÇÕES FINAIS}

Constatou-se também que a adequação da metodologia desenvolvida para a avaliação do desenvolvimento local municipal e seus aspectos da sustentabilidade, através do cálculo do IDSM, pode oferecer significativas contribuições para o processo de tomadas de decisão pelos gestores públicos e na implementação de políticas públicas para o desenvolvimento local sustentável dos municípios do Estado de Mato Grosso do Sul.

Apesar da redução do número de variáveis disponíveis em âmbito municipal para o cálculo do IDSM, os resultados deste trabalho devem contribuir para a ampliação do debate sobre o desenvolvimento local sustentável municipal e estabelecer diferenças entre os níveis de sustentabilidade entre os municípios, ao mesmo tempo em que pode subsidiar os gestores públicos em suas decisões.

Os resultados encontrados para os municípios do Estado apontam para problemas relacionados a aspectos institucionais como infraestrutura nos domicílios e alunos na universidade. 
Isso fez com que os municípios fossem classificados em termos de níveis de sustentabilidade como em Alerta e Aceitável, não havendo nenhum município nos extremos de Crítico nem Ideal.

Os municípios classificados com os piores resultados em Alerta foram: Japorã, Paranhos, Tacuru, Coronel Sapucaia, Miranda, Jaraguari, Nioaque e Sete Quedas. Já os municípios com melhores resultados foram: Campo Grande, Dourados, Três Lagoas, Chapadão do Sul, São Gabriel do Oeste, Maracaju, Corumbá, Costa Rica e Nova Andradina.

\section{REFERÊNCIAS}

BARTELMUS, P. Towards a framework for indicators of sustainable development. Working Papers Series n. 7. New York: United Nations, 1994.

BUARQUE, S. C. Construindo o desenvolvimento local sustentável: metodologia de planejamento. Rio de Janeiro: Garamond, 2008.

HAK, T. INDI-LINK: Review Report on the State-of-the-Art in Sutainable Development Indicators. Annex 1: Sustainable Development Indicators Initiatives by Selected International Organizations, 2007. Disponível em: <http://www.indilink.net/images/stories/Download/ indilink_deliverable_d_1.1_annexes.pdf>. Acesso em: 10 ago. 2014.

INSTITUTO BRASILEIRO DE GEOGRAFIA E ESTATÍSTICA (IBGE). Indicadores de Desenvolvimento Sustentável: Brasil 2012. Rio de Janeiro: IBGE, 2014.

JOLLANDS, N. How to aggregate sutainable development indicators: a proposed frameword and its application. International Journal Agriculture Resources, Governance and Ecology, v. 5, n. 1, p. 18-34, 2006.

MARTINS, M. F.; CÂNDIDO, G. A. Índice de Desenvolvimento Sustentável para Municípios (IDSM): metodologia para análise e cálculo do IDSM e classificação dos níveis de sustentabilidade - uma aplicação no Estado da Paraíba. João Pessoa: Sebrae, 2008.

NADER, M. R. et al. Environment and sustainable development indicators in Lebanon: a practical municipal level approach. Ecological Indicators, v. 8, n. 3, p. 771-777, 2008. Disponível em: <http:// www.sciencedirect.com/science/journal/1470160X/44/supp/C>.

NOURRY, M. Measuring sustainable development: some empirical evidence for France from eight / alternative indicators. Ecological Economics, v. 67, n. 3, p. 441-456, 2008. Disponível em: <http://www.sciencedirect.com/science/journal/09218009/77/supp/C>.

NAÇÕES UNIDAS (NU). Indicators of sustainable development: guilidelines and methodologies. 3. ed. New York: NU, out. 2007.

2001.

Indicators of Sustainable Development: Framework and Methodologies. New York: NU,

ROLDÁN, A. B.; SALDÍVAR-VALDÉS, A. Proposal and application of a Sustainable Development Index. Ecological Indicators, v. 2, n. 3, p. 251-256, 2002. Disponível em: <http://www.sciencedirect. com/science/journal/1470160X/44/supp/C>.

RONCHI, E. et al. A system oriented integrated indicator for sustainable development in Italy. Ecological Indicators, v. 2, n. 1, p. 197-210, 2002. Disponível em: <http://www.sciencedirect. com/science/journal/1470160X/44/supp/C>.

WAQUIL, P. et al. Avaliação de desenvolvimento territorial em quatro territórios rurais no Brasil. Redes, v. 15, n. 1, p. 104-127, 2010. 


\section{Sobre os autores:}

Daniel Massen Frainer: Doutorado em Economia pela Universidade Federal do Rio Grande do Sul (UFRGS). Mestrado em Engenharia de Produção e Graduação em Ciências Econômicas pela Universidade Federal de Santa Maria (UFSM). Coordenador de Economia de Estatística do Governo do Estado de Mato Grosso do Sul. Atualmente desenvolve projetos relacionados com o tema de Matriz de Insumo-Produto Regional na Universidade Estadual de Mato Grosso do Sul (UEMS), Bolsista PNPD-Capes. E-mail: danielfrainer@gmail.com

Celso Correia de Souza: Professor Titular do Doutorado em Meio Ambiente e Desenvolvimento Regional. Universidade Anhanguera-Uniderp (UNIDERP), Campo Grande, Mato Grosso do Sul, Brasil. E-mail: celso939@gmail.com

José Francisco Reis Neto: Professor colaborador do Doutorado em Meio Ambiente e Desenvolvimento Regional. Universidade Anhangura-Uniderp (UNIDERP), Campo Grande, Mato Grosso do Sul, Brasil. E-mail: jfreisneto@terra.com.br

Raul Asseff Castelão: Doutorando do Programa de Pós-Graduação em Meio Ambiente e Desenvolvimento Regiona. Faculdade Salesiana de Santa Tereza (FSST), Corumbá, Mato Grosso do Sul, Brasil. E-mail: raulassefcastelao@gmail.com 